\title{
Overjet problems at the growing child, case report using the twin block appliance
}

\author{
Muhammad H. Achmad*
}

\section{Abstract}

Objective: Using a twin block appliance to treat overjet problem in growing child.

Methods: Twin-block consists of maxilla and mandible blocks bite with retention $0.7 \mathrm{~mm}$ adams clasped on permanent first molars and $0.9 \mathrm{~mm}$ balls clasped placed on maxillary and mandibular incisors embrasures. Passive maxillary bow labial used for anterior retention and control the incisors proclination. Lateral expansion of maxillary arch was achieved by expansion screw-controlled once a week. The patient was instructed to use appliance for 24 hours a day, wear and remove the maxillary and mandibular twin-block by himself, activate the screw expansion twice a week and followed up every three weeks. The patient was also instructed to oral hygiene maintenance especially around the covered acrylic plate region.

Results: Patient's profile shapes was improved after 7 months treatment. Crowding mandible reduced by proclination of mandibular incisor, normal occlusion with optimum overbite as well as overjet could be achieved.

Conclusions: Twin block appliances was successfully treat the overjet problem in this patient.
Department of Pediatric, Faculty of Dentistry, Hasanuddin University Makassar, Indonesia
${ }^{*}$ Correspondence to: Muhammad Harun Achmad, Department of Pediatric, Faculty of Dentistry, Hasanuddin University Makassar, Indonesia harunachmader@gmail.com

Keywords: Overjet, Growing child, Children malocclusion, Twin Block.

Cite this Article: Achmad MH. 2017. Overjet problems at the growing child, case report using the twin block appliance. Journal of Dentomaxillofacial Science 2(1): 49-53. D0l: 10.15562/jdmfs.v2i1.454

\section{Introduction}

Malocclusion involves complex influence between genetic factors and environmental factors. Inherited mandible growth pattern (genetic) leads the chin to grow downward and backward which can be na predisposing factor of malocclusion. Environmental factors such as a habit, oral habit, the movement of soft tissue and early tooth loss can contribute to malocclusion. Sucking persistently (thumb sucking) over 4 years old related to high prevalence of increased overjet and the relationship class II of caninus and molar. ${ }^{1,2}$ Unbalanced force of oral soft tissue, such as tongue thrust habit, low tonicity of muscle lips and bad tongue rest position can lead to the displacement tooth position due to change in balance. Early tooth loss, especially in maxillary primary molar causes mesial drifting on maxillary permanent molar, hence it can also be a local factor affecting the development of malocclusion class II. ${ }^{2}$ Parents' education, birth rate, social and economy status, effect of breastfeeding duration to sucking habit and diet influence affects teeth malocclusion on growing children. ${ }^{3,4}$

Overjet is a horizontal relationship between maxillary and mandibular incisors. Normal range of overjet is $2-4 \mathrm{~mm}$. Increased overjet is marked by protrusion of maxillary incisors. Overjet size over $6 \mathrm{~mm}$ can impact on psychology and social burden in children as a result of the aesthetic on child's face profile shapes. Increased overjet related to class II malocclusion is usually accompanied by class II skeletal sagittal relationship and retrognathia of the mandible. ${ }^{1,3,4}$

Overjet which is part of class II division 1 malocclusion is related to two fundamental things about the diagnosis of malocclusion. Increased overjet is the main characteristics of the diagnosis of class II division 1 malocclusion. Further, an increased overjet as the characteristics of the diagnosis of class II malocclusion is a risk factor of trauma in maxillary incisor. This problem based on a critical assessment result of several randomized clinical studies in last 15 years about the treatment results of class II malocclusion which explains that increased overjet could define class II malocclusion. The claim of several research reveals that prevention of trauma on maxillary incisors is seen as an indication for early treatment of class II malocclusion..$^{3,4}$

In Angle classification based on occlusal pattern, increased overjet which is a characteristic of the diagnosis of class II division 1 malocclusion is related to maxillary skeletal relationship, anterior teeth protrusion and mandibular skeletal retrusion. Increased in overjet can be found as an orofacial disharmony on someone's face who has thumb or lips sucking habit that is usually accompanied by lips incompetence. That increased overjet is a significant characteristic of occlusal that is related 
to dentoskeletal of class II division 1 malocclusion. ${ }^{2,5,6}$

\section{Eeffects of Increased Overjet}

\section{a. Anterior Trauma ${ }^{1,2,4}$}

Clinical problems related to increased overjet is an association with the incidence of trauma on the permanent incisors during growing age. Increased prevalence of trauma on maxillary incisors is correlated with the class II division 1 malocclusion (increased in overjet). In a systematic review of the relationship between increased overjet and trauma on maxillary incisors, it was found that children with overjet over $3 \mathrm{~mm}$ had two fold risk of trauma on the anterior teeth compared to children with smaller overjet. Children about the risk of trauma on maxillary incisors increased by $13 \%$ along with increased overjet. There are statistically significant relationship between the increased overjet and trauma on teeth, children with class II malocclusion have $64 \%$ opportunities on teeth trauma. Teeth trauma observed such as tooth fracture occurs three times higher on child with the case of increased overjet, so it is important to seek malocclusion prevention and treatment. Educational counseling is needed to reduce bad habits that can cause malocclusion (overjet increasing) as an effort to minimize the prevalence of teeth trauma in children.

\section{b. Tissue damage $\mathbf{e}^{1,9,10}$}

Teeth fracture is related to overjet greater than $6 \mathrm{~mm}$. Incidence of incisors trauma were high in boys than girls (boys:girls, 2:1). A child with overjet greater than $8 \mathrm{~mm}$ tends to show lost bone support compared to someone with overjet in the normal range. Periodontal damage occurs along with increased age, periodontal ligament becomes less resistant to atypical pressure lips related with normal lips closure difficulty. Harmonization of the incisors relationship during growing age can contribute to prevent teeth migration in older age. Increased overjet is related to increased plaque accumulation and gingivitis.

\section{Social effects ${ }^{1,9,11}$}

Several studies show the social impact on a child who has excessive overjet. Overjet increasing condition is considered as unattractive look, overjet greater than 6-9 $\mathrm{mm}$ results mockery, rejection, a joke, pity, curious reaction and unnatural gaze from other people around patients. Protrusive upper teeth (convex face) is being the reason of closure lips difficulty, could be a shameful thing for many children. It has been the reason for parents to seek orthodontic treatment for their child. Reasons for those conditions are: aesthetic, increased risk of maxillary incisors trauma, likely increased periodontal problems and decreased chewing ability.

\section{d. Symptoms of temporomandibular joint disorder ${ }^{1,9,10}$}

Increased overjet is related with the signs and symptoms of temporomandibular disorder (TMD). Overjet $>5 \mathrm{~mm}$ is correlated with TMD. The impact of increased overjet on temporomandibular joint's function occurs because of less contact on incisors, much mandible movement and pressure on the muscle mastication. The larger the space on centric occlusion (teeth relation when the jaw on the rest position) the more maximum contact exists between the cusp (occlusion habit regardless of the location condyle) and this results to one of the symptoms of temporomandibular joint function called the orthopedic instability.

Orthopedic instability caused by centric occlusion deflection is one of the important thing on TMD etiologic factor. Changed muscle tension and tone is influenced by the amount of centric occlusion space to maximum contact between the cusp, especially if it is asymmetric. Patients with large overjet show significant difference on condyle position on mandibular fossa when in centric occlusion position and centric relations compared to patients with normal overjet. Practitioners should give special attention on temporomandibular joint status of patient with large overjet.

\section{Management}

There are two options to lower increased overjet: Labial segment retraction of maxilla and labial segment development of mandible. Preferred choice will depend on factors relating to the skeletal pattern, soft tissue, as well as the age of patients. Options to reduce the increased overjet can be by using removable appliance, functional appliance which aims to change dental and skeletal relation, fixed orthodontic appliance for tipping, and bodily movement by repositioning the jaw through orthognathic surgery. ${ }^{1,8,9}$

\section{Management with the Twin-Block functional appliance}

Twin-block functional appliance developed by Clark is functional appliance that has been widely used for class II malocclusion treatment especially in efforts to reduce large overjet. This appliance can be used in a long time that allows movement on the mandible, easy to use, patients can be cooperative. However to get maximum treatment results depends good cooperation of each patients. 

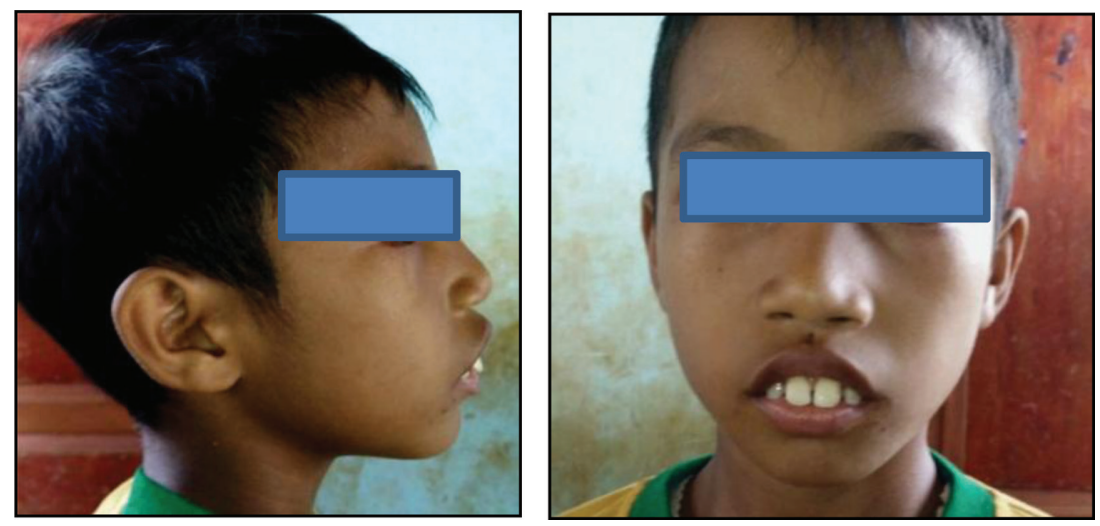

Figure 1 Clinical photograph of patient's face profile before treatment
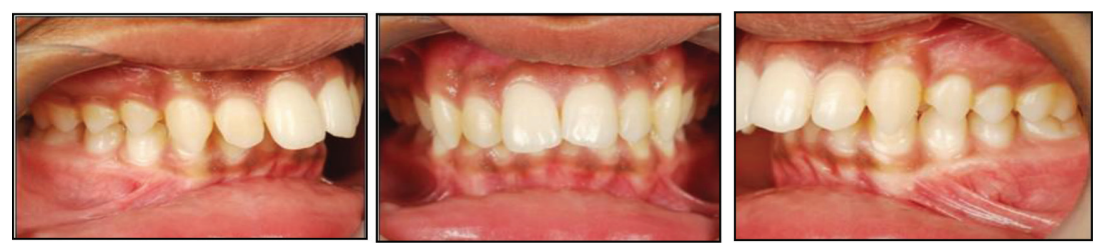

Figure 2 Intraoral photograph before treatment

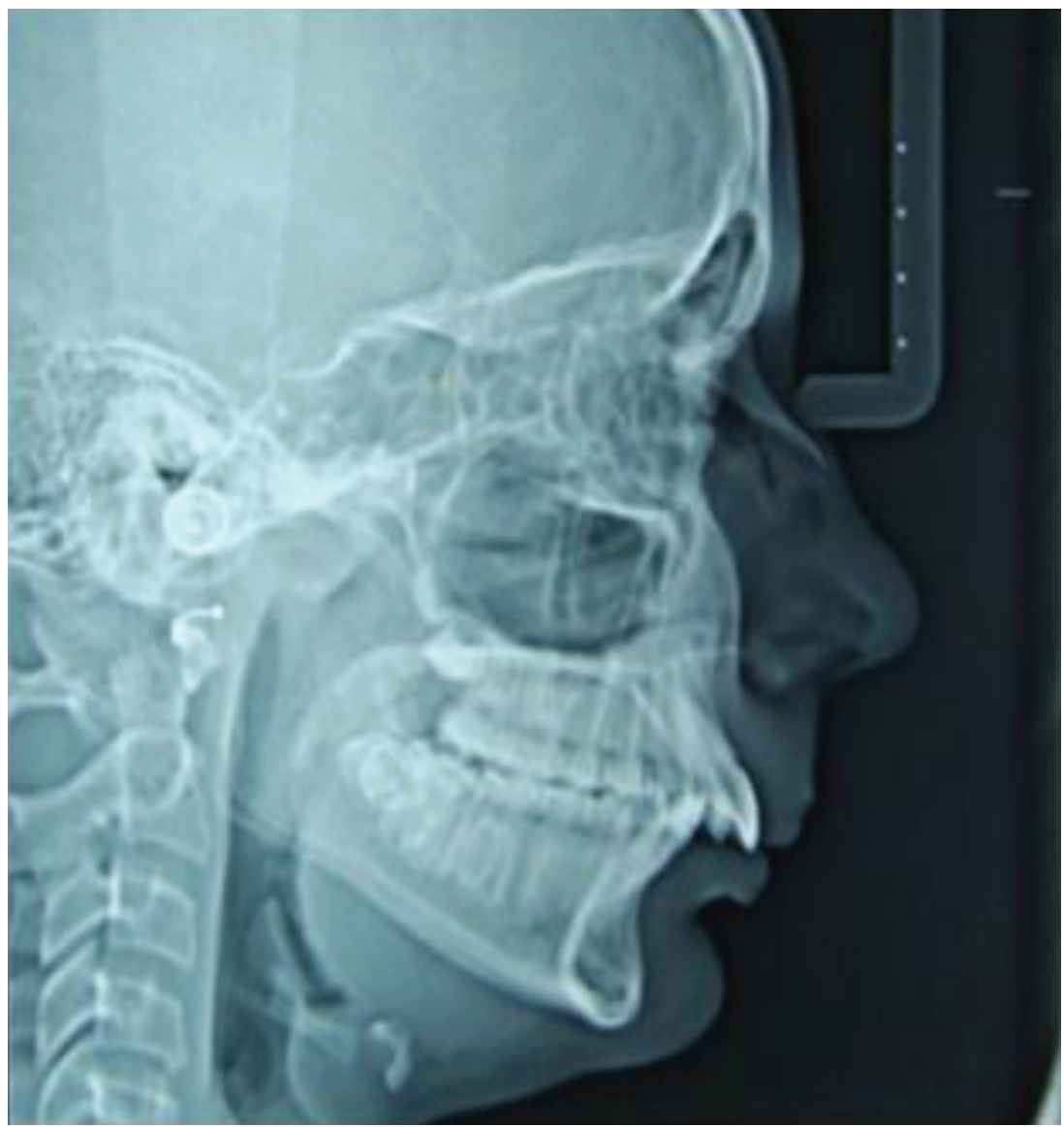

Figure 3 Cephalometric radiographs

This appliance is widely used for child treatment especially at the growing age. Using twin-block functional appliance shows that the early treatment can reduce overjet effectively, change form of skeletal pattern, as well as increase in child's psychology such as: confidence along with aesthetic improvement on children's face significantly.

\section{Case Report}

A 12 year old boy came to Dental Hospital, Department of Pediatric, Faculty of Dentistry, Hasanuddin University, Makassar, Indonesia with chief complaint that front teeth of upper jaw more forward figure 1 and 2. Treatment plan: planning treatment on anteroposterior plane with removable appliance (twin-block removable). Treatment purpose of this case was to correct relation between maxilla and mandible become class I.

Twin-block consists of maxilla and mandible blocks bite with retention $0.7 \mathrm{~mm}$ adams clasped on permanent first molars and $0.9 \mathrm{~mm}$ balls clasped placed on maxillary and mandibular incisors embrasures. Passive maxillary bow labial used for anterior retention and control the incisors proclination. The plane side sharp interlocked around $70^{\circ}$ to the occlusal plane. Lateral expansion of maxillary arch was achieved by expansion screw-controlled once a week. Block reactivation was done as needed. The patient was instructed to use appliance for 24 hours a day (except for the contact sports and swimming).

\section{Treatment Progress}

Each stage progress of this functional treatment was achieved because of patient's cooperation figure 4. These treatments completed for 7 months. Maxillary incisor retroclined $9^{\circ}$ while the mandibular incisor proclinated $4^{\circ}$. This achievement resulted in reduced overjet figure 5. Patients were instructed to wear and remove the maxillary and mandibular twin-block by himself and instructed to activate the screw expansion twice a week and followed up every three weeks. The patient was also instructed to oral hygiene maintenance especially around the covered acrylic plate region. Patients needed to clean plate or twin block appliance after eating because the food will usually accumulate under the plate, cleaning thoroughly using a toothbrush and toothpaste then rinsed with cold water.

\section{Treatment Results}

After functional correction by positioning mandible forward, patient's profile showed harmonization increased of the face balance figure 4 normal occlusion with optimum overbite and overjet could be achieved figure 5. Patient was satisfied with facial profile, which has changed from convex to normal. 

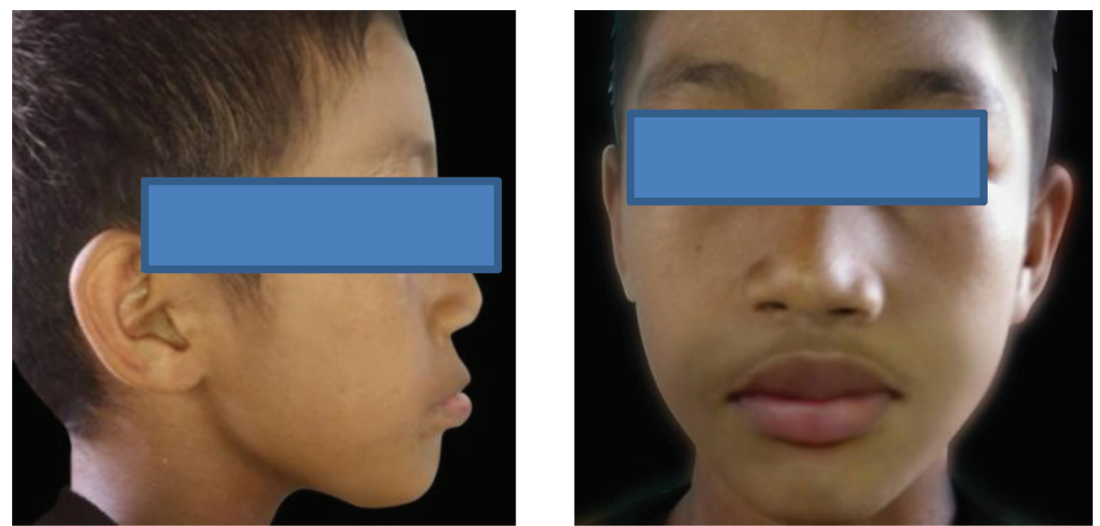

Figure 4 Clinical photograph of patient's face profile after treatment
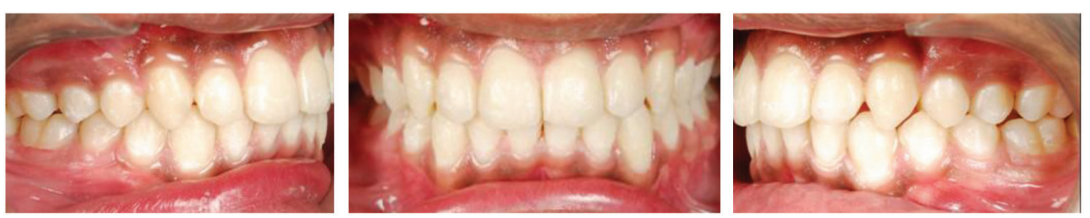

Figure 5 Intraoral clinical photograph after treatment
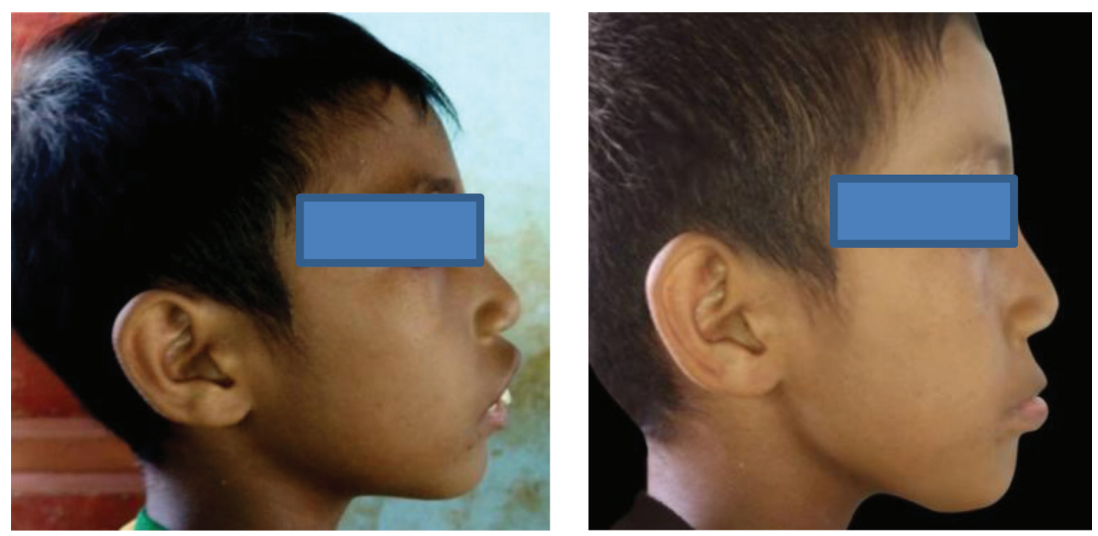

Figure 6 Clinical photograph changes in patient's face profile
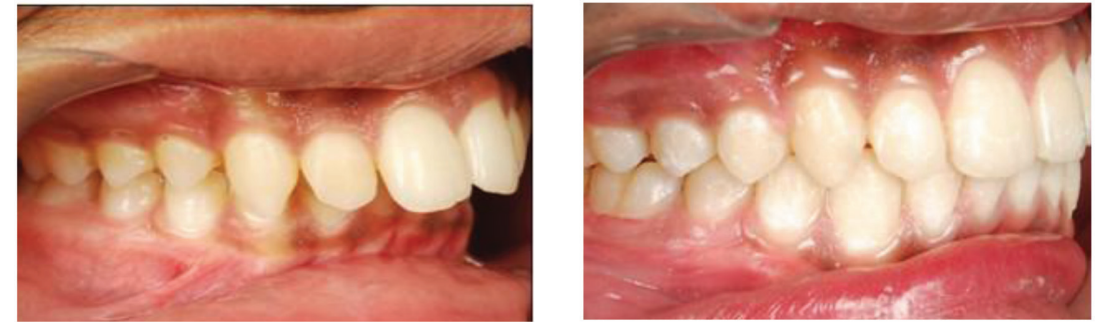

Figure 7 Intraoral clinical photograph before and after treatment

Cephalometric analysis showed mandible position forwardly, maxilla growth restriction and good interincisal angle.

Treatment results showed patient's profile shapes has improved after treatment figure 4. Crowding mandible reduced by proclination of mandibular incisor. At the end of treatment showed class I incisors, canine and molar relation figure 5. Overbite and overjet was reduced. Developmental changes is shown in figure 6 and table 1.
Tabel 1 Cephalometric tracing changes before and after treatment

\begin{tabular}{lccc}
\hline & Before & Rata2 & After \\
\hline$<$ SNA $\left(^{*}\right)$ & 76 & 82 & 79 \\
$<$ SNB $\left(^{*}\right)$ & 71 & 80 & 74 \\
$<$ ANB $\left(^{*}\right)$ & 5 & 2 & 3 \\
1 ke NA $(\mathrm{mm})$ & 9 & $4 \mathrm{~mm}$ & 6 \\
$<1$ ke NA $\left(^{*}\right)$ & 36 & 22 & 30 \\
1 ke NB $(\mathrm{mm})$ & 3 & $4 \mathrm{~mm}$ & 5 \\
$<1$ ke NB $\left(^{*}\right)$ & 26 & 25 & 23 \\
$<$ Pg ke NB $(\mathrm{mm})$ & 3 & $2 \mathrm{~mm}$ & 3 \\
$<1$ ke $1\left(^{*}\right)$ & 113 & 131 & 124 \\
$<$ Okl ke SN $\left(^{*}\right)$ & 25 & 14 & 19 \\
$<$ GoGn ke SN $\left(^{*}\right)$ & 37 & 32 & 35 \\
\hline
\end{tabular}

\section{Discussion}

Characteristics class II skeletal and dentoalveolar malocclusion in patients with large overjet become important reason of early treatment necessity to control amount and direction of mandible growth using ideal functional appliance on this case. Malocclusion prevention and treatment study, as well as health care policy should be included in a corrective treatment for malocclusion. Educational program will ease the guide and reduce bad habits which can cause malocclusion (increased overjet). Teeth trauma in children is often associated with aesthetic, psychological, social problems and therapy. Therefore, it requires more efforts to health improvement and prevention strategies implementation to reduce the level of teeth trauma on preschool children. ${ }^{4,7,12}$

Twin-block functional appliance has several advantages including the fact that it is well accepted by the patient, strong, repaired easily and suitable for mixed dentition period and permanent teeth. Size of this appliance is easy touse by the patient so that speech interference could be minimized. Treatment purposes can be achieved due to the patient's cooperation. Reduction overjet with functional appliance results to better patient's confidence and minimize the risk of trauma on the maxillaryincisors. ${ }^{8,12,13}$

The selection of functional appliance depends on several factors, such as patient's age and the level of cooperative, habit factors as well as the availability of laboratory facilities. During the treatment, the SNA value was reduced $1^{\circ}$ while the $\mathrm{SNB}$ value was increased $1^{\circ}$. As a consequence ANB value decreased $2^{\circ}$ resulted in class I skeletal pattern. 
Maxillary incisor inclination reduced to 1160 . Mandibular incisor was proclinated 4o vertical.

Proclinating mandibular incisor used capping acrylic was reported to reduce amount of proclination maxillary incisor. Mandibular molar moved to mesial. It has been shown in the literature that the functional appliance does not make long term skeletal changes and most of all effects result in dentoalveolar. However, it can be seen in this case that the functional appliance allows fixed appliance stage to achieve good results. In this case, patient's profile is improved. Arrangement have been made to control patient's development regularly during treatment stage. Similarly, use of retention such as hawley retainer required to ensure stability. ${ }^{12,13}$

\section{Conclusion}

Twin block appliances was successfully treat the overjet problem in this patient.

\section{Conclusion}

Twin block appliances was successfully treat the

\section{References}

1. Baccetti T, Giuntini V, Vangelisti A, et al. Diagnostic performance of increased overjet in class II division 1 malocclusion and incisor trauma. Prog Orthod 2010;11: 145-150

2. Turasi B, Demirkaya AA, Biren S. Comparison of increased overjet cases and controls: normative data for condylar positions. J Oral Rehabil 2007;34: 129-135.
3. Jabbar NSA, Bueno ABM, Silva PED, et al. Bottle feeding, increased overjet and class 2 primary canine relationship: is there any association?. Braz Oral Res 2011;25: 331-337.

4. Antunes LAA, Gomes IF, Almeida MH, et al. Increased overjet is a risk factor for dental trauma in preschool children. Indi J Den Res 2015;26: 356-360.

5. Wagner Y, Weltzien RH. Occlusal characteristic in 3 years old children-results of a birth cohort study. BMC Oral Health 2015;15: 1-6.6.

Antoniou C dan kawan-kawan. Class II Division 1 Malocclusions-early diagnosis \& management part. Australian Society of Orthodontist. www.aso.org.au; 2010. p. 1-4.

7. Ramesh N, Guruanthan D, Khartikeyyan SA. Association of nonnutritive sucking habits and Malocclusion: a cross-sectional study. Int J Pedodont Rehab 2016;1; 15-18.

8. Denny JM, Weiskircher MA, Dorminey JC. Anterior open bite dan overjet treated with camouflage therapy. Am J Orthop 2007;131: 670-678.

9. O’Brien K, Wright J, Conboy F, et al. Early treatment for Class II division 1malocclusion with the twin-block appliance: A multi-center, randomized, controlled trial. Am J Orthod Dentofacial Orthop 2009;135: 573-579.

10. Puri T, Patel D. Skeletal Discrepancy Correction In Class II Div 1 Malocclusion Using Fixed Twin Blocks. IOSR JDMS 2014;13: 65-67.

11. Sood S. Treatment of Class II division 1 Malocclusion in a non growing patient. VJO 2010.

12. Gupta S, Kumar-Jindal S, Bansal M, et al. Prevalence of traumatic dental injuries and role of incisal overjet and inadequate lip coverage as risk factors among 4-15 years old government school children in BaddiBarotiwala Area, Himachal Pradesh, India. Med Oral Patol Oral Cir Bucal 2011;16: 960-965.

13. Saltaji H, Flores-Mir C. The relationship between vertical facial morphology and overjet in untreated Class II subjects. Angle Orthod 2012:82:432-440.

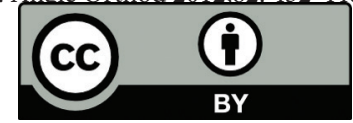

This work is licensed under a Creative Commons Attribution 